\title{
Piogranuloma estéril idiopático em cão: relato de caso
}

Idiopathic sterile pyogranuloma in dog: case report

\section{Piogranuloma estéril idiopático en perros: relato de caso}

\section{Fernando Malagutti Cunha; ${ }^{1}$ Lúcia Maria Guedes Silveira; ${ }^{2}$ Priscyla Taboada Dias da Silva; ${ }^{3}$ Cássio Ricardo Auada Ferrigno ${ }^{4}$}

Hospital Veterinário do Instituto de Ciências da Saúde da Universidade Paulista, Campus Tremembé (UNIP). São Paulo, SP, Brasil

\section{Resumo}

Objetivo: Relatar um caso de enfermidade dermatológica piogranulomatosa estéril idiopática em cão, detalhandose exames complementares realizados e terapia empregada. Descrição: Cocker Spaniel, três anos de idade, apresentando lesão solitária, ulcerada e indolor, próxima ao lábio superior esquerdo. Diagnóstico estabelecido após análises dermato-histológicas seriadas e exclusão de agentes infecciosos como causa do processo. Conclusões: Trata-se de cutaneopatia incomum no cão, cujo diagnóstico final baseia-se na dermatopatologia. A excisão cirúrgica completa de lesão única é curativa.

Palavras-chave: Dematopatias. Granuloma piogênico. Cães

\footnotetext{
'Professor Adjunto de Clínica Médica de Pequenos Animais. Departamento de Medicina Veterinária do Instituto de Ciências da Saúde da UNIP. CRMV-SP 9509 ${ }_{2}^{2}$ Professora Adjunta de Clinica Cirúrgica de Pequenos Animais. Departamento de Medicina Veterinária do Instituto de Ciências da Saúde da UNIP. CRMV-SP 8573 ${ }^{3}$ Patologista do Serviço de Anatomia Patológica Veterinária - Histopet ${ }^{\star}$ CRMV-SP 11732

${ }^{4}$ Professor Doutor do Departamento de Cirurgia da Faculdade de Medicina Veterinária e Zootecnia da Universidade de São Paulo. CRMV-SP 6835
} 


\section{Introdução}

Lesões dermatológicas granulomatosas/ piogranulomatosas podem ser ocasionadas por agentes infecciosos (bacterianos, fúngicos ou virais), parasitas ou corpos estranhos, entretanto, nem todos os casos apresentam causa determinável..$^{1-3} \mathrm{O}$ piogranuloma cutâneo estéril possui caráter idiopático, sendo pouco observado no cão., ${ }^{2,-6} \mathrm{Tal}$ desordem pode ocorrer em qualquer faixa etária e em ambos os sexos, tendo sido descrita no Dachshund, ${ }^{1}$ Boxer, Collie, Weimaraner, ${ }^{2}$ Golden Retriever ${ }^{4}$ Rottweiler $^{7}$ e Labrador. ${ }^{8}$

As alterações cutâneas apresentam-se solitárias ou múltiplas e afetam a cabeça, os pavilhões auriculares e as extremidades de membros. ${ }^{1,2,4,6,8}$ Placas, pápulas ou nódulos estão usualmente presentes, os quais, por vezes, sofrem ulceração. ${ }^{2,6,9}$ Não há envolvimento sistêmico. ${ }^{2,6} \mathrm{O}$ estabelecimento do diagnóstico final exige correta interpretação de informações colhidas na anamnese, exame físico, cultivo e dermatopatologia. ${ }^{1,2,4,6}$ As opções terapêuticas compreendem excisão de lesão única extirpável, ${ }^{2,6}$ ou utilização de fármacos imunomoduladores quando o tratamento cirúrgico for inviável. ${ }^{1,5,7}$ Acredita-se que aproximadamente $60 \%$ dos casos requerem corticoterapia sistêmica em dose imunossupressora por tempo indefinido. ${ }^{6} \mathrm{O}$ presente caso é relatado objetivando registrar uma cutaneopatia incomum no cão, exames auxiliares requeridos no diagnóstico e modelo terapêutico adotado.

\section{Relato do caso}

Um Cocker Spaniel, macho, três anos de idade, foi atendido no Hospital Veterinário da Universidade Paulista, São Paulo, SP, com queixa de lesão cutânea ulcerada, indolor e não pruriginosa, situada em adjacência de lábio superior esquerdo há 20 dias. $\mathrm{O}$ cão era domiciliado e adequadamente imunizado. Não havia menção à presença de contactantes, ocorrência de trauma ou uso de medicamentos. Ao exame físico, constatou-se boa condição corpórea geral, integridade de linfonodos periféricos, normotermia

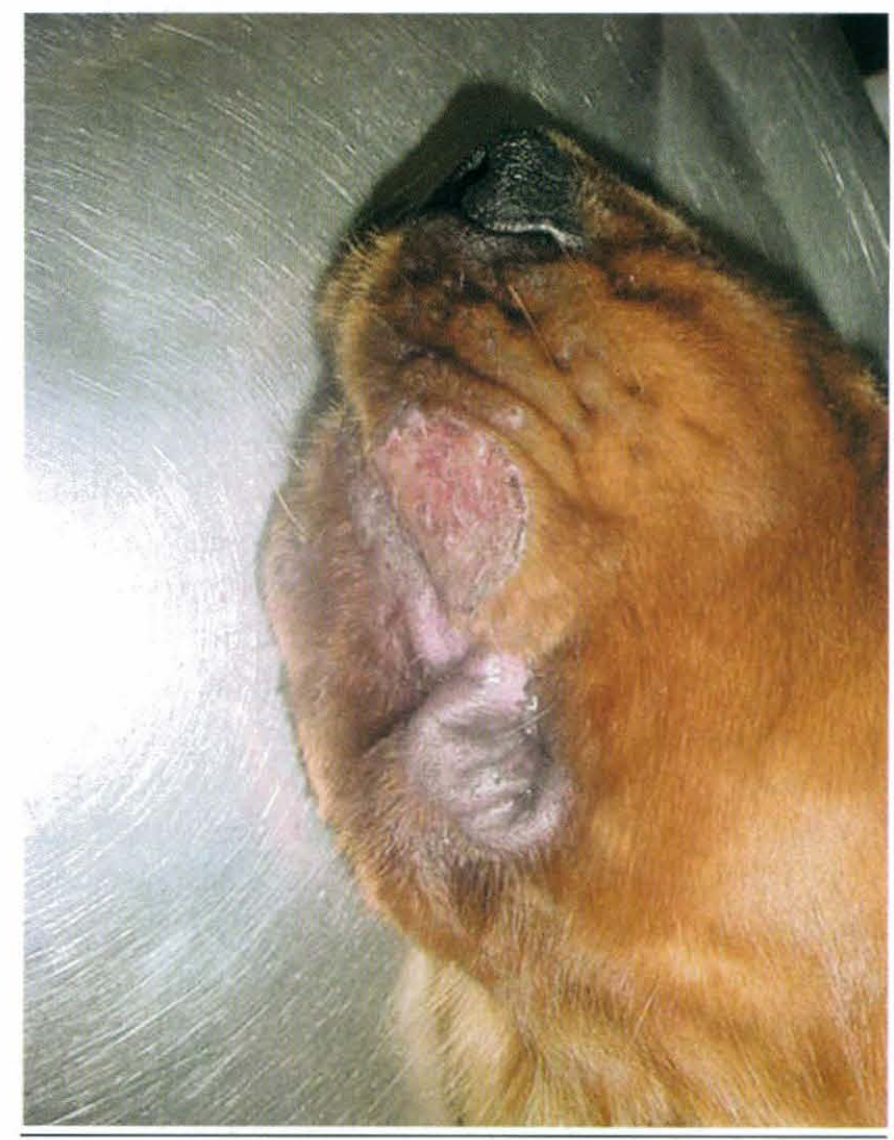

$\overline{\text { Figura } 1 \text { - Piogranuloma estéril idiopático. Observar lesão }}$ dermatológica ulcerada próxima ao lábio superior

e presença de lesão dermatológica solitária, ulcerada, de dois centímetros de diâmetro, localizada próxima ao lábio superior esquerdo (Figura 1). Os demais sistemas orgânicos avaliados não apresentaram alterações dignas de nota.

Realizou-se biópsia incisional, detectando-se reação inflamatória piogranulomatosa perianexal e ausência de agentes infecciosos concomitantes ou sinais de malignidade em secção histológica corada 


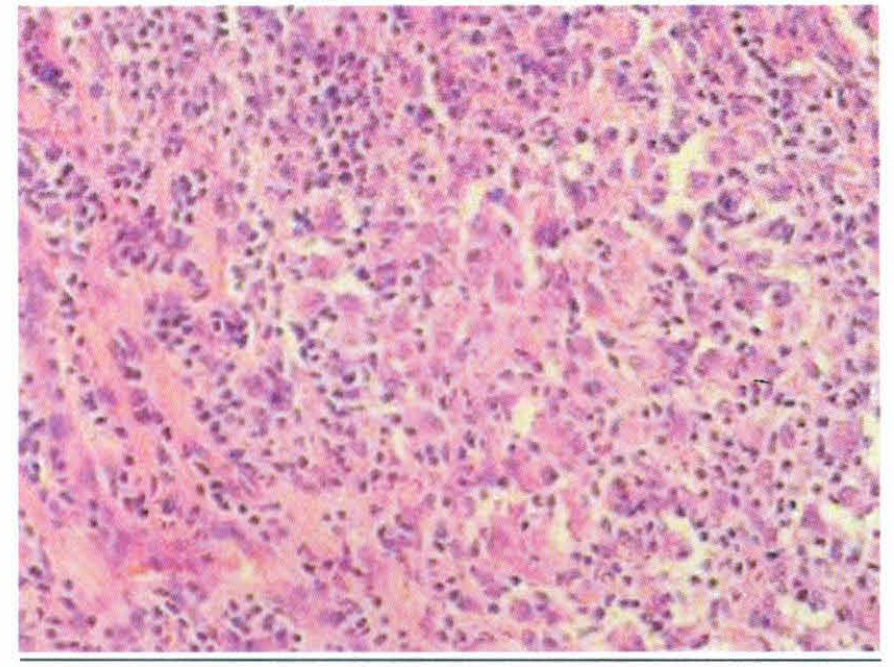

Figura 2 - Fotomicrografia de corte histológico de pele, demonstrando infiltrado inflamatório piogranulomatoso (HE 400X)

em hematoxilina/eosina (Figura 2). O cultivo de fragmentos cutâneos obtidos dessa mesma lesão mostrou-se negativo quanto ao crescimento de bactérias ou fungos causadores de micoses profundas. Procedeu-se à excisão cirúrgica completa da lesão e à nova análise dermatopatológica em hematoxilina/ eosina, a qual revelou alterações micromorfológicas similares às anteriormente verificadas. Amostras deste tecido foram também submetidas às técnicas de coloração de Zielhn-Neelsen e Groccot, apresentando-se negativas para presença de micobactérias e microorganismos fúngicos, respectivamente, descartando-se, então, a participação de agentes infecciosos no processo. A interpretação do painel de dados obtidos permitiu estabelecer o diagnóstico de piogranuloma cutâneo estéril idiopático. O cão recebeu acompanhamento clínico com retornos periódicos por 60 dias após a cirurgia, não havendo indícios de recidiva ou complicações associadas ao procedimento operatório executado. Nenhuma terapia farmacológica foi instituída.

\section{Discussão}

A ausência de agentes causais específicos, tal como verificado no presente caso, permite classificar o piogranuloma estéril como enfermidade dermatológica idiopática. ${ }^{2,4-6}$ Trata-se de uma cutaneopatia incomum, porém já descrita em raças distintas do cão deste relato. ${ }^{1,2,4,7,8}$

$\mathrm{Na}$ avaliação física do mencionado animal, foi constatada lesão dermatológica solitária, ulcerada e indolor situada na face e ausência de comprometimento sistêmico, sintomatologia também observada nos casos previamente documentados. ${ }^{1,2,4,6,8,9}$

O diagnóstico baseia-se na exclusão de possíveis agentes etiológicos, principalmente os infecciosos, associando-se dados obtidos na anamnese, exame físico-dermatológico e testes complementares apropriados. ${ }^{1,2,4,6,7}$ Cabe ressaltar que no cão atendido, a dermatopatologia novamente realizada com técnicas destinadas à identificação de patógenos específicos, bem como o cultivo de fragmentos desta mesma amostra, revelaram-se negativos para a presença de microorganismos, alicerçando o diagnóstico de piogranuloma cutâneo estéril idiopático.

A excisão cirúrgica como terapia única demonstrou eficácia na resolução da enfermidade, fato já registrado na literatura pertinente. ${ }^{2,6}$

\section{Conclusões}

- Considerando-se a casuística dermatológica do Hospital Veterinário da Universidade Paulista e dados anteriormente publicados, concluiu-se que o piogranuloma estéril idiopático é uma dermatopatia incomum no cão;

- O diagnóstico definitivo repousa na dermatopatologia e no descarte de possíveis agentes causais utilizando-se testes destinados a esse fim;

- A extirpação cirúrgica de lesão solitária excisável representa opção terapêutica efetiva, sendo curativa nos casos que exibem esse tipo de manifestação clínica. 


\section{Abstract}

Objective: To report a case of a dermatological disease referred as idiopathic sterile pyogranuloma in a dog, detailing the ancillary tests performed and the selected therapy. Description: Cocker Spaniel, three years old, presenting with an isolated, ulcerated and painless lesion close to the left upper lip. Diagnosis made after serial dermatohistological analyses and exclusion of infectious agents as cause of the process. Conclusions: It is an uncommon cutaneous disease in the dog, with a final diagnosis based on dermatopathology. The radical surgical excision of the single lesion is curative.

Keywords: Skin disease. Granuloma, pyogenic. Dogs.

\section{Resumen}

Objetivo: Relatar un caso de enfermedad dermatológica piogranulomatosa estéril idiopática en perro, detallándose exámenes complementarios realizados y su terapia aplicada. Descripción: Cocker Spaniel, tres años de edad, presentando lesión aislada, ulcerada e indolora, próxima al labio superior izquierdo. Diagnóstico fundamentado después de estudios dermatopatológicos en serie y exclusión de agentes infecciosos como causa del proceso. Conclusiones: Se trata de dermatopatía incomún en perros, cuyo diagnóstico final se basa en la dermatopatología. La excisión quirúrgica completa de la lesión única es curativa.

Palabras-clave: Dermatopatía. Granuloma piogénico. Perros.

\section{Referências}

1. CARLOTTI, D. N. et al. Aspects cliniques et histopathologiques des pseudo-neoplasmes cutanés chez le chien, le chat, le cheval et les ruminants. I. Granulomes et pyogranulomes. Le Point Veterinaire, v. 31, p. 45-55, 2000.

2. PANIC, R. Sterile pyogranulomatous and granulomatous disorders of dogs and cats. In: KIRK, R. W.; BONAGURA, J. D. Current veterinary therapy. XI. Small animal practice. Philadelphia: Saunders, 1992. p. 536-539.
3. SCOTT, D. W.; NOXON, J. O. Sterile sarcoidal granulomatous skin disease. Canine Practice, v. 15 , p. 11-18, 1990.

4. HOUSTON, D. M. et al. A case of cutaneous sterile pyogranuloma/granuloma syndrome in a Golden Retriever. Canadian Veterinary Journal, v. 34, p. 121-122, 1993.

5. ROTHSTEIN, E. et al. Tetracycline and niacinamide for the treatment of sterile pyogranuloma/granuloma 
syndrome in a dog. Journal of the American Animal Hospital Association, v. 33, p. 540-543, 1997.

6. SCOTT, D. W. et al. Miscellaneous skin diseases. In: Small animal dermatology. Philadelphia: Saunders, 2001. p. 1125-1183.

7. BARRET, S. J. et al. Challenging cases in internal medicine: what's your diagnosis? Veterinary Medicine, v. 93, p. 35-44, 1998.
8. COLLINS, B. K. et al. Idiopathic granulomatous disease with ocular adnexal and cutaneous involvement in a dog. Journal of the American Veterinary Medical Association, v. 201, p. 313 316, 1992.

9. CARPENTER, J. L. et al. Idiophatic periadnexal multinodular granulomatous dermatitis in twentytwo dogs. Veterinary Pathology, v. 24, p. 5-10, 1987. 